Pramāna, Vol. 8, No. 4, 1977, pp. 328-334, Printed in Indja.

\title{
Density of states of the binary alloy in the coherent potential approximation
}

\author{
A PRASANNA KUMAR* \\ Physics Department, Bangalore University, Bangalore 56c001 \\ * Present Address: Guest Staff Member, Department of Theoretical Physics, \\ University of New Castle, New Castle Upon Tyne, United Kingdom.
}

MS received 16 September 1976; in revised form 23 December 1976

\begin{abstract}
The density of states of an electron in a binary alloy in the tight binding model is calculated in the single site coherent potential approximation (CPA) as a function of the concentration and the site energy difference. The fluctuations in the site energies due to the random environment is taken into account approximately by giving width to the site energy probability distribution function, which is normally a sum of two delta functions with proper weight factor.
\end{abstract}

Keywords. Binary alloy; disordered systems; coherent potential approximation.

\section{Introduction}

Understanding the properties of alloys has become a topic of considerable interest in solid state physics in recent times. The first two approximations used in the study of electronic properties of alloys are rigid band and virtual crystal approximations. Because of the unsatisfactory nature of these approximations in explaining the observed properties, attempts were made to approach the problem differently. Recently the averaged $T$-matrix approximation (ATA) introduced by Korringa (1958) and Beeby (1964) and the coherent potential approximation (CPA) introduced by Soven (1967) and Taylor (1967) have proved to be reasonably successful models. Both the ATA and CPA are based on multiple scattering theory formalism originally introduced by Foldy (1945) and Lax (1952). These two methods have been employed to study both model and realistic systems.

In the single site description of CPA one regards the total scattered wave to be composed of contributions from each atom while the effective wave incident on a given atom excludes the contribution of that atom. This contribution is obtained as a product of the atomic $t$-matrix and the effective wave. Velicky et al (1968) have put the CPA on a more formal footing and have established that CPA is the best single site description available. The CPA is the simplest self-consistent approximation which gives the first several moments of the density of states correctly (Schwartz and Siggia 1972). CPA and ATA become exact in the calculation of the average single particle Green function of the electron in 
the Anderson model when the site energy is distributed randomly with CauchyLorentzian distribution. This result was obtained by Prasanna Kumar and Baskaran (1973) for both ATA and CPA and independently by Bishop (1973) for the CPA case. The CPA has been investigated extensively by several authors and the comprehensive reviews by Elliot et al (1974), Ehrenreich and Schwartz (1976) and Yonezawa and Morigaki (1973) contain details of the numerous application of CPA and ATA which exist in the literature.

In this paper we present a detailed CPA calculation for the density of states of a modified binary alloy model in which the site energy associated with each component is itself a random variable with a Cauchy-Lorentzian distribution. The site energy distribution is thus a sum of two Cauchy-Lorentzian distributions weighted by the concentrations rather than a sum of two $\delta$-functions as usually assumed. It is suggested that this modification accounts phenomenologically for the fluctuations in the environment of a given site which must occur in any real system.

\section{The model for binary alloy}

We consider the 2-component alloy to be described in the tight binding representation. The one-electron Hamiltonian in the tight binding representation is given by,

$$
\begin{aligned}
H & =\sum_{n} \epsilon_{n} C_{n}^{+} C_{n}+\sum_{m \neq n} V_{m n} C_{n}^{+} C_{n} \\
& \equiv V+H_{0}
\end{aligned}
$$

where $C_{n}^{+}$and $C_{n}$ are the creation and annihilation operators for the electron in the Wannier orbital centred at the site $n . V$ is the diagonal part and $H_{0}$ is the non-diagonal part of the Hamiltonian. The diagonal elements $\epsilon_{n}$ are the random atomic energy levels which assume values $\epsilon_{1}{ }^{0}$ and $\epsilon_{2}{ }^{0}$ and with respective probabilities $a_{1}$ and $a_{2}$, depending on whether an atom of type 1 or 2 occupies. the site $n$. Since we are essentially dealing with a one body problem, in the absence of any magnetic interaction and magnetic field we omit the spin index in eq. (1). The random variables $\epsilon$ should be characterised by a suitable probability distribution $P(\epsilon) . \quad V_{n m}$ is the interaction or the hopping matrix element between sites $n$ and $m$. The hopping matrix elements are assumed to be independent of the alloy configuration. The operator $H_{0}$ may be interpreted as the Hamiltonian of the pure crystal for which

$$
\epsilon_{1}{ }^{0}=\epsilon_{2}{ }^{0}=0 \text {. }
$$

We also assume that $V_{n m}$ is non-zero only for the nearest neighbours and that $V_{n m}$ is non-random. The distribution of the constituent atoms of the alloy is assumed to be statistically independent so that the probability of finding any one of the types of atoms 1,2 on an arbitrary chosen site $n$ is $a_{1}, a_{2}$ respectively. The joint probability distribution $P\left(\left\{\epsilon_{n}\right\}\right)$ is given by

$$
P\left(\left\{\epsilon_{n}\right\}\right)=\prod_{n} p\left(\epsilon_{n}\right)
$$

where $P\left(\epsilon_{n}\right)$ is expressed as

$$
P\left(\epsilon_{n}\right)=\sum_{s=1}^{2} a_{s} \delta\left(\epsilon_{n}-\epsilon_{s}{ }^{0}\right)
$$


with

$$
a_{1}+a_{2}=1,
$$

where $a_{1}$ is the concentration of type 1 atom and $a_{2}\left(=1-a_{1}\right)$ is the concentration of type 2 atom. So far it has been generally assumed that the energy of the orbital can take any one of the energies of the constituent atoms. But, in reality, due to the random environment there will be a spread in the orbital energies. So, in general, the distribution function $P(\epsilon)$ will not be a sum of $\delta$-functions but will be a sum of functions peaked around $\epsilon_{3}^{0}$ 's. Thus it takes into account the spread in the energies around the mean values $\epsilon_{i}{ }^{0}$. This approach, to a certain extent, effectively takes into account the fluctuations in the environment of a given site as well as some other perturbations which gives rise to energy spread. For mathematical convenience we assume that $P(\epsilon)$ is the sum of Cauchy-Lorentzian distributions $P_{L}(\varepsilon)$ given by,

$$
P_{L}(\epsilon)=\frac{1}{\pi}\left(\frac{\Gamma}{\epsilon^{2}+\Gamma^{2}}\right) \text {. }
$$

The expression for $P(\epsilon)$ becomes

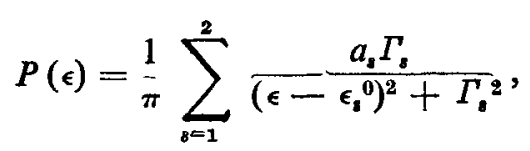

where $\Gamma_{1}$ and $\Gamma_{2}$ are the half width of the distributions.

The density of states of the binary alloy can in principle be calculated by using the CPA.

The electronic properties of the binary alloy can be discussed most simply in terms of the single particle Green function $\langle n|G(E)| m\rangle$ which is the configuration space representation of the operator

$$
G(E)=(E-H)^{-1}=\left(E-H_{0}-V\right)^{-1}
$$

$G(E)$ depends on the occupancies of all sites of the alloy. This dependence of $G(E)$ is removed by performing an ensemble average. We also define,

$$
G^{0}(E)=\left(E-H_{0}\right)^{-1}
$$

$G(E)$ may be used to define the self-energy $U(E)$,

$$
\langle G(E)\rangle=(E-H-U(E))^{-1}
$$

$U(E)$ is in general complex and non-Hermitian. Here " $\langle\ldots\rangle$ " denotes the averaging with respect to the random site energies. Our main task then is to calculate $\langle G(E)\rangle$ which is done here by employing the self-consistent CPA.

\section{Density of states of the two component (binaty) alloy: CPA model}

For convenience we choose $\epsilon_{1}{ }^{0} \equiv C / 2$ and $\epsilon_{2}{ }^{0} \equiv-C / 2$. The two distributions are centred at energies $C / 2$ and $-C / 2$ from the origin as shown in figure 1 . In this model, the variation in $C$ corresponds to the variation in site energy and the variation in the height of the distribution corresponds to the change in the concentration. By varying $C$ the overlap of the two distributions can be altered. 


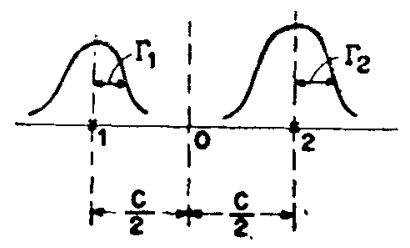

Figur: 1. Two-component (binary) alloy.

Thus we specify the alloy completely by $\Gamma, a$ and $C$. These are our input variables. What we need is $\langle G\rangle$ and it is calculated self-consistently in the CPA as follows. Consider the Dyson equation,

$$
G=\bar{G}+\bar{G} T \bar{G}
$$

where $T$ is the total scattering matrix ( $T$-matrix) defined as

$$
T=\sum_{n} T_{n}, T_{n}=t_{n}\left(1+\bar{G} \sum_{n \neq m} T_{m}\right) .
$$

Here

$$
t_{n}=\frac{\epsilon_{n}-U}{-\left(\epsilon_{n}-U\right) \tilde{G}}
$$

$\mathrm{j}$ s the $T$-matrix corresponding to the $n$th site. We want to find $G$ self-consistently such that $\langle T\rangle=0$ (which implies that $\langle G\rangle=\bar{G}$ ). In the single site CPA we write,

$$
\left\langle t_{n} \sum_{n \neq m} T_{m}\right\rangle \approx\left\langle t_{n}\right\rangle \sum_{m \neq n}\left(T_{m}\right) .
$$

Therefore

$$
\langle T\rangle=\sum_{m}\left(T_{m}\right) \approx \Sigma\left\langle t_{n}\right\rangle\left(1+\langle G\rangle \sum_{m \neq n}\left\langle T_{m}\right\rangle\right) .
$$

In the CPA the density of states of the binary alloy model is calculated by expliciting the condition $\left\langle t_{n}\right\rangle=0$. We therefore have,

$$
\left\langle t_{n}\right\rangle=\int_{-\infty}^{\infty} \frac{\epsilon-U}{1-(\epsilon-U)\langle G\rangle} P(\epsilon) d \epsilon=0 .
$$

The explicit dependence of $U$ and $G$ on $E$ is omitted for brevity. In the case of the binary alloy we consider $P(\epsilon)$ to be given by,

$$
P(\epsilon)=\frac{1}{\pi}\left(\frac{a_{1} \Gamma_{1}}{\left(\epsilon+\frac{C}{2}\right)^{2}+\Gamma_{1}^{2}}+\frac{a_{2} \Gamma_{2}}{\left(\epsilon-\frac{C}{2}\right)^{2}+\Gamma_{2}^{2}}\right)
$$

where $a_{1}$ and $a_{2}$ are the concentrations of the two species and $C$ is the separation between the two sites energies. Substituting for $P(\epsilon)$ eq. (15), we get,

$$
\left\langle t_{n}\right\rangle=\frac{1}{\pi} \int_{-\infty}^{+\infty} \frac{(\epsilon-U)}{1-(\epsilon-U)\langle G\rangle}\left(\frac{a_{1} \Gamma_{1}}{\left(\epsilon+\frac{C}{2}\right)^{2}+\Gamma_{1}^{2}}+\frac{a_{2} \Gamma_{2}}{\left(\epsilon-\frac{C}{2}\right)^{2}+\Gamma_{2}^{2}}\right) d \epsilon=0 .
$$


The above integral can be performed by the contour integration method (Lloyd 1969). Using the general analytic properties of $U$ and $G$, we get

$$
\begin{aligned}
& U^{2}\langle G\rangle+U\left(1-i \Gamma_{1}\langle G\rangle-i \Gamma_{2}\langle G\rangle\right)+i\left(\Gamma_{1} a_{1}-\Gamma_{1}-\Gamma_{2} a_{2}\right) \\
& \quad+\left(\frac{C}{2}-C a_{2}-\Gamma_{1} \Gamma_{2}\langle G\rangle-\frac{i C\langle G\rangle}{2}\left[\Gamma_{1}-\Gamma_{2}\right]-\frac{C^{2}\langle G\rangle}{4}\right)=0
\end{aligned}
$$

This is a quadratic equation which can easily be solved for $U$. We consider the semicircular band case, for which

$$
G_{0}=2 / B^{2}\left(E-\sqrt{E^{2}-B^{2}}\right) .
$$

Now $U$ is also given by (Browers 1971)

$$
U=E-\frac{1}{\langle G\rangle}-\frac{\langle G\rangle B^{2}}{4},
$$

where $2 B$ is the band width.

Using (18) and (19) we can get an expression for $G$.

$$
\begin{aligned}
& \langle G\rangle^{3}\left(\frac{B^{2}}{4}\right)+\langle G\rangle^{2} B^{2}\left(i \Gamma_{1}+i \Gamma_{2}-2 E\right) \\
& \quad+\langle G\rangle\left[4 B^{2}-4 E\left(i \Gamma_{1}+i \Gamma_{2}\right)-4 \Gamma_{1} \Gamma_{2}+2 C i\left(\Gamma_{1}-\Gamma_{2}\right)+B^{2}-C^{2}\right] \\
& \quad+\left(4 i \Gamma_{2}-4 i a_{2} \Gamma_{1}+i a_{2} \Gamma_{2}-4 E+4 C a_{2}-2 C\right)=0 .
\end{aligned}
$$

The density of states is given by,

$$
\rho(E)=-\frac{1}{\pi} \vartheta m\langle G(E+i o)\rangle \text {. }
$$

Figures (3), (4), (5) give the density of states of the binary alloy computed from (20). For comparison we have reproduced the curves of Schwartz et al (1971) (figure 2) where they have calculated the density of states in the CPA and ATA. The

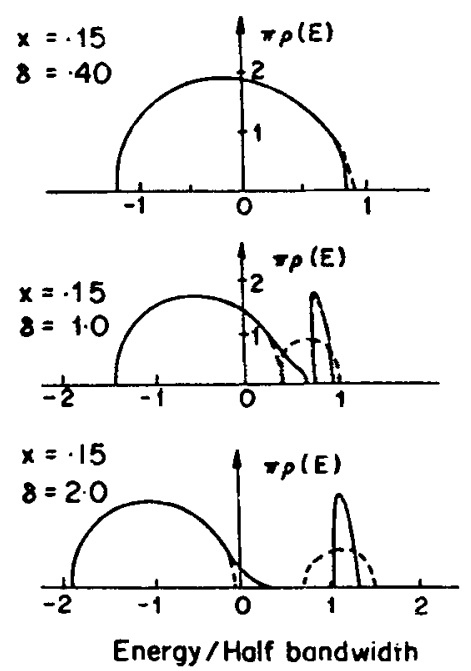

(a)

Figure 2. Comparison of the density of states calculated in the coherent rotential approximation and the averaged $T$-matrix approximations (from Schwartz et al 1971). 

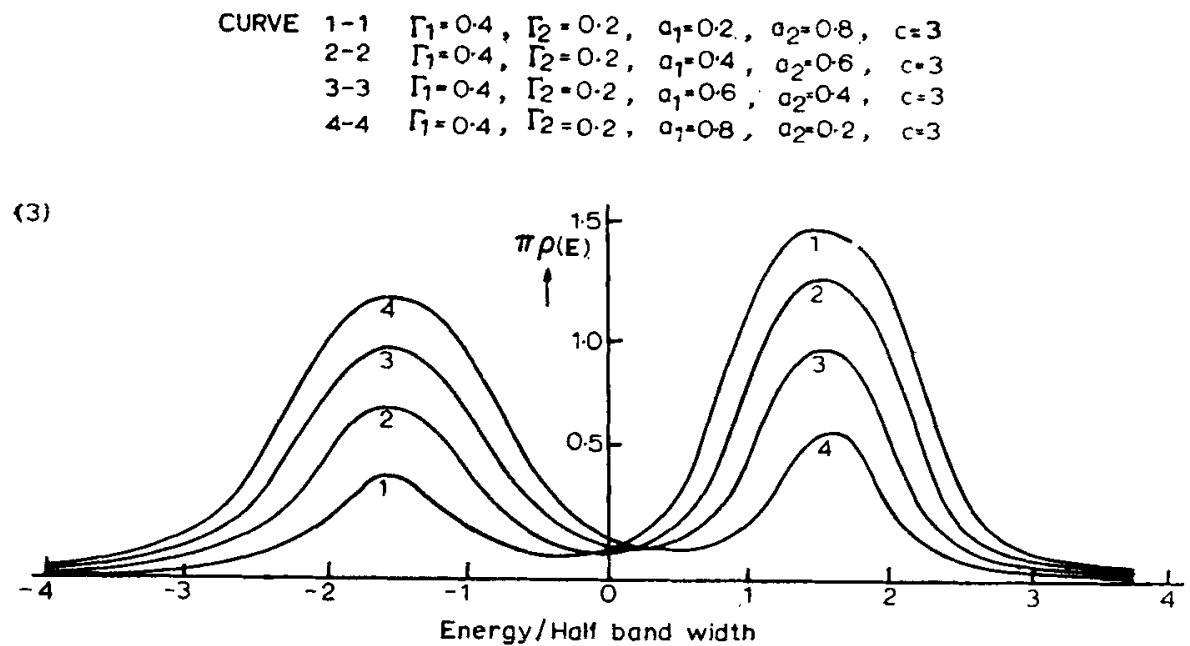

$$
\text { CURVE } \begin{aligned}
1-1 & \Gamma_{1}=0.4, \Gamma_{2}=0.2, a_{1}=0.2, a_{2}=0.8, c=4 \\
2-2 & \Gamma_{1}=0.4, \Gamma_{2}=0.2, a_{1}=0.2, a_{2}=0.8, c=3 \\
3-3 & \Gamma_{1}=0.4, \Gamma_{2}=0.2, a_{1}=0.2, a_{2}=0.8, c=2
\end{aligned}
$$

(4)

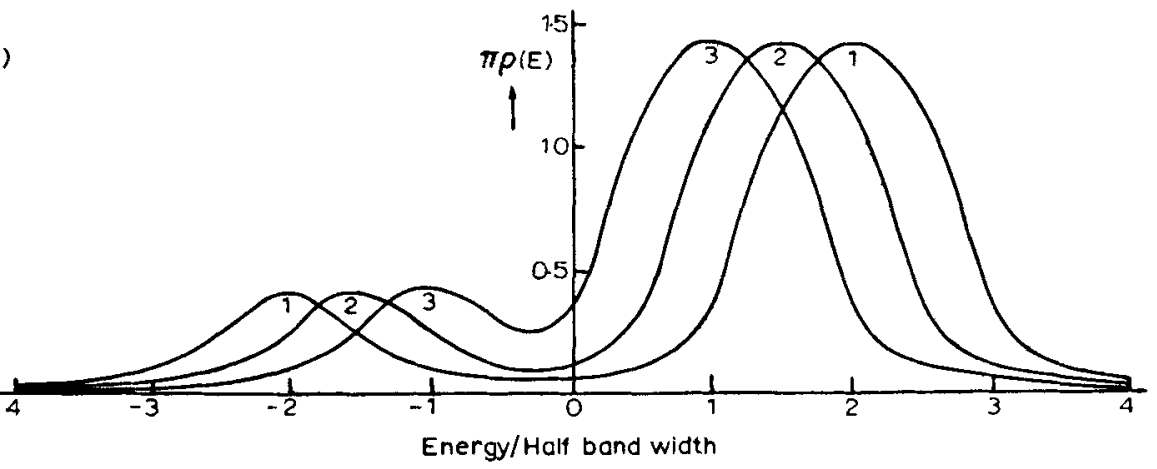

$$
\begin{aligned}
\text { CURVE } 1-1 & \Gamma_{1}=0.4, \Gamma_{2}=0.4, a_{1}=0.5, a_{2}=0.5, c=4 \\
2-2 & \Gamma_{1}=0.2, \Gamma_{2}=0.2, a_{1}=0.5, a_{2}=0.5, c=4 \\
3-3 & \Gamma_{1}=0.2, \Gamma_{2}=0.2, a_{1}=0.5, a_{2}=0.5, c=2
\end{aligned}
$$

15)

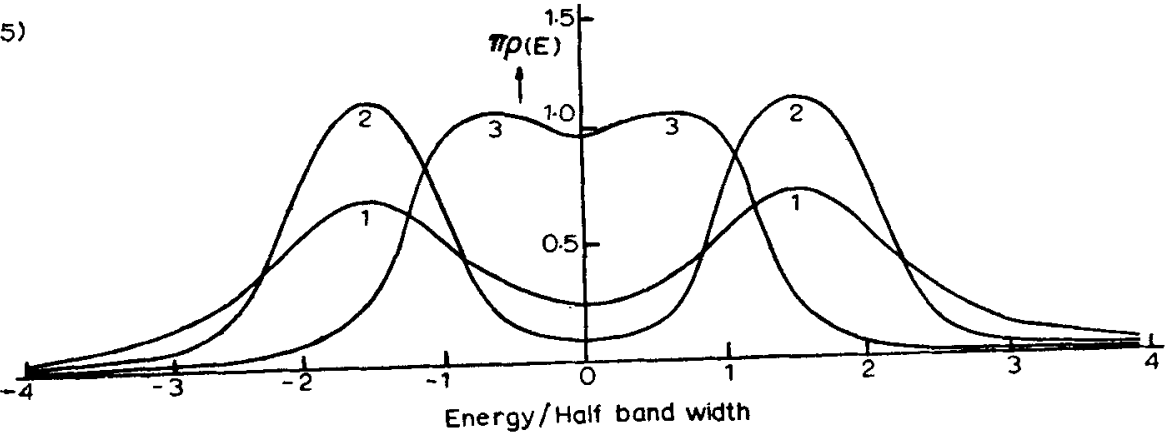

Figures 3-5. Density of states of the binary alloy in the CPA. 
curves of Schwartz et al display the extended region of the density of states well but fail to produce the tails where the states are localized. Our model gives the profile of the density of states better as indicated by the figures. The long tails might be associated with the nature of the distribution chosen (CauchyLorentzian). The band edges display the tails in our model and hence we can say that the model approaches the real system more closely at least with regard to the density of states. The model thus displays the features of the Mott-CFO model better. We cannot however say that the present model has incorporated all the important and relevant features of the alloy, since the tails in real alloys arise from cluster effects which lie beyond GPA proper.

In figures 3-5 we have plotted the density of states of the binary alloy in the CPA. In figure $3 \Gamma_{1}$ and $\Gamma_{2}$, the widths of the distributions are kept constant (but different) and the concentrations are allowed to vary. As $a_{1}$ changes from 0.2 to 0.8 the shape of the density of states curve changes (curve 1.1 goes over to curve 4-4). The curves demonstrate that our model predicts the expected variation in the density of states profile when the concentration changes.

In figure $4 \Gamma_{1}$ and $\Gamma_{2}$ are different but held constant. The concentrations of $a_{1}$ and $a_{2}$ also do not change. The parameter that changes is the separation of the two distributions. When the two distributions are far apart (compared to the width) there is a dip in the centre of the band. This is because the distributions are far apart and do not overlap very much. As the overlap increases the nature of the dip in the centre of the band changes and there will now be more states in the centre of the band. Also the peak begins to shift towards the centre.

In figure 5, we have considered a special case. The widths as well as the concentrations of the species are maintained constant and equal (curves 1 and 2). As the width of the distribution increases the overlap becomes more and the curve gets flattened. If the separation is also not too much the dip in the centre of the band becomes less as displayed by curve 3 . Our model of the binary alloy accounts phenomenologically for the fluctuations in the environment of a given site which will occur in a real system.

\section{References}

Beeby J L 1964 Proc. R. Soc. (London) $\mathbf{A 2 7 9} 82$

Bishop A R 1973 Phil. Mag. 27651

Browers F 1971 J. Phys. C4 773

Elliott R J, Krumhansl J A and Leath P L 1974 Rev. Mod. Phys, 46465

Foldy L L 1945 Phys. Rev. 67107

Korringa J 1958 J. Phys. Chem. Solids 7252

Lax M 1952 Phys. Rev. 85621

Lloyd P 1969 J. Phys. C2 1717

Prasanna Kumar A and Baskaran G 1973 J. Phys. C6 1399

Schwartz L and Siggia E 1972 Phys, Rev. B5 383

Schwartz L, Browers F, Vedyayev A V and Ehrenreich H 1971 Phys, Rev. B10 3383

Seven P 1967 Phys. Rev. 156809

Taylor D W 1967 Phys. Rev. 1561017

Velicky B, Kirkapatrick S and Ehrenreich H 1968 Phys. Rev. 175747

Yonezawa F and Morigaki K 1973 Sup. Prog. Theor. Phys. No. 53

Ehrenreich H and Schwartz L M 1976 Solid State Phys. 31149 (The author thanks the referee for bringing this reference to his attention) 